
\title{
is Research Square \\ Regorafenib Combined With Sintilimab Versus Regorafenib as Second-line Treatment for Advanced Hepatocellular Carcinoma
}

\author{
Jingjun Huang \\ Guangzhou Medical University Second Affiliated Hospital
}

\section{Yongjian Guo}

Guangzhou Medical University Second Affiliated Hospital

Wensou Huang

Guangzhou Medical University Second Affiliated Hospital

\section{Zining Xu}

Guangzhou Red Cross Hospital

\section{Liteng Lin}

Guangzhou Medical University Second Affiliated Hospital

Jingwen Zhou

Guangzhou Medical University Second Affiliated Hospital

Licong Liang

Guangzhou Medical University Second Affiliated Hospital

\section{Yaqin Zhang}

Fifth Affiliated Hospital of Sun Yat-sen University

Juan Zhou

Guangzhou Medical University Second Affiliated Hospital

Mingyue Cai

Guangzhou Medical University Second Affiliated Hospital

Kangshun Zhu ( $\sim$ zhksh010@163.com )

Guangzhou Medical University Second Affiliated Hospital https://orcid.org/0000-0001-5142-010X

\section{Research Article}

Keywords: Liver cancer, Regorafenib, Sintilimab, Combined treatment, Second-line treatment, Overall survival, Progression-free survival, Objective response rate, Neutrophil-to-lymphocyte ratio, Prognostic factor

Posted Date: September 15th, 2021

DOI: https://doi.org/10.21203/rs.3.rs-872090/v1

License: (c) (1) This work is licensed under a Creative Commons Attribution 4.0 International License. Read Full License 


\section{Abstract}

Purpose: To evaluate the safety and efficacy of regorafenib combined with immune checkpoint inhibitor sintilimab (rego-sintilimab) as second-line treatment for advanced hepatocellular carcinoma (HCC) patients who failed prior sorafenib or lenvatinib.

Methods: This retrospective study evaluated consecutive patients with advanced HCC who received rego-sintilimab (rego-sintilimab group) or regorafenib alone (regorafenib group) as second-line treatment from January 2019 to December 2020. Adverse events, objective response rate (ORR), progression-free survival (PFS), and overall survival (OS) were compared between the two groups.

Results: Eighty-three patients were included: 48 received rego-sintilimab and 35 received regorafenib. Rego-sintilimab group had higher ORR (33.3\% vs $14.3 \%, P=.049$ ), longer PFS (median, 5.1 vs 3.0 months; $P=.001$ ), and better OS (median, 13.3 vs 9.1 months; $P=.001$ ) than regorafenib group. Regorafenib alone, Child-Pugh $\mathrm{B}$, and neutrophil-tolymphocyte ratio (NLR) $>3.5$ were independent prognostic factors for poor OS in uni- and multi-variable analyses. Subgroup analyses showed that, in patients with Child-Pugh A (16.4 vs 11.5 months; $P=.005)$, Child-Pugh B ( 8.8 vs 6.4 months; $P=.032$ ), or NLR $\leq 3.5$ (16.3 vs 11.5 months; $P=.012$ ), rego-sintilimab group had significantly better median OS than regorafenib group, whereas median OS was not significantly different between the two groups in patients with NLR $>3.5$ (8.4 vs 7.0 months; $P=.288$ ). The incidences of grade $3 / 4$ adverse events were similar between the two groups ( $39.4 \%$ vs $34.1 \% ; P=.445)$.

Conclusion: Rego-sintilimab was tolerable and led to better OS than regorafenib as second-line treatment for advanced HCC patients, especially in those with NLR $\leq 3.5$.

\section{Introduction}

Despite recent improvements in surveillance programs, $50-75 \%$ of patients with hepatocellular carcinoma (HCC) are diagnosed at an advanced stage [1]. Multi-kinase inhibitor sorafenib and lenvatinib are the first-line treatments for advanced HCC, as recommended by updated Barcelona Clinical Liver cancer (BCLC) treatment algorithms [1]. However, these two drugs only result in a median progression free survival (PFS) of 3.7-7.3 months [2, 3], and a second-line treatment is needed after disease progression. Multi-kinase inhibitor regorafenib [4], cabozantinib [5], and VEGFR-2 inhibitor ramucirumab [6] are appropriate for pretreated patients with advanced HCC. Nonetheless, these second-line treatments only resulted in an objective response rate (ORR) of $4-11 \%$ and a median OS of $8.5-10.6$ months versus a median OS of 7.3-8.0 months for placebo. Considering the low ORR and modest OS improvement, a more effective second-line treatment is needed.

Immunotherapy with immune checkpoint inhibitors (ICI) is currently a possible second-line option. Phase $1 / 2$ data highlighted durable responses with $\mathrm{ICl}$, such as nivolumab and pembrolizumab, for HCC patients who failed prior sorafenib treatment [7, 8]; however, phase 3 data of pembrolizumab has failed to demonstrate improved OS compared to placebo in second-line setting [9]. Since ICI monotherapy was not significantly effective, combination of $\mathrm{ICl}$ and tyrosine-kinase inhibitors or VEGF inhibitors is being widely considered [10]. Regorafenib is potent VEGFR2/3 inhibitor, which may lead to promising ORR in combination with ICl immunotherapy [11]. An HCC model has shown that the combination of regorafenib and ICl could increase intratumoral CD8 + T-cell infiltration by normalizing the tumor vasculature [12], and subsequently improve the efficacy of ICI immunotherapy. 
Recently, ICI sintilimab combined with VEGF inhibitor (bevacizumab biosimilar) showed a significant OS benefit versus sorafenib in the first-line setting for Chinese HCC patients [13]. Sintilimab has a high programmed death-1 (PD-1) receptor occupancy rate (>95\%) [14] and long occupancy duration on circulating T cells [15], which indicates that it may have a powerful anti-tumor immune effect. Considering the above characteristics of regorafenib and sintilimab, their combination (rego-sintilimab) may have an advantage over the current standard second-line regorafenib monotherapy. Therefore, this study aimed to investigate the safety and efficacy of rego-sintilimab, in comparison to regorafenib alone, for advanced HCC patients in a second-line setting.

\section{Materials And Methods}

\section{Patient Selection}

We reviewed the electronic medical records of consecutive patients with advanced HCC who receive rego-sintilimab or regorafenib after disease progression on sorafenib or lenvatinib treatment from January 2019 to December 2020 at our institution. Patients were assigned to receive rego-sintilimab or regorafenib according to patients' decision under the attending physician's suggestion. This retrospective study was approved by our institutional review board. Written informed consent was obtained from every patient prior to treatment.

HCC was diagnosed according to the European Association for the Study of Liver and American Association for the study of Liver Disease guidelines [16]. The inclusion criteria for the study population were as follows: (a) age between 18 and 75 years, (b) Eastern Cooperative Oncology Group (ECOG) performance status of 0 or 1, (c) BCLC stage C HCC, (d) Child-Pugh A or B liver function, and (e) radiographic disease progression on first-line treatment with sorafenib or lenvatinib. Patients were excluded from this study if they (a) had previously received targeted therapy besides to sorafenib or lenvatinib monotherapy, (b) had previously received immunotherapy, (c) currently had or had a history of malignant tumors in addition to HCC, (d) had severe medical comorbidities including severe organ dysfunction and coagulation disorders, such as creatinine $\geq 1.5$ upper limit of normal or international normalized ratio $\geq 1.5$, or (e) had a follow-up less than 3 months.

\section{Data collection}

The baseline characteristics were collected within one week before the initiation of rego-sintilimab or regorafenib. Inflammation-based prognostic scores neutrophil-to-lymphocyte ratio (NLR) and platelet-to-lymphocyte ratio (PLR) were calculated based on the baseline data. NLR was calculated as the neutrophil count divided by the lymphocyte count and PLR as the platelet count divided by the lymphocyte count [17].

All the dynamic CT or MR imaging analyses, including tumor size, tumor number, macrovascular invasion, extrahepatic metastasis, and treatment response, were conducted independently by two diagnostic radiologists who were blinded to treatment allocation and clinical information. When there was any ambiguity, the final determination was made by consensus.

\section{Treatment protocol and adjustment}

Regorafenib was initially prescribed $120 \mathrm{mg} /$ day, which might be adjusted to $160 \mathrm{or} 80 \mathrm{mg} /$ day according to the patients' tolerance, during weeks 1-3 of each 4 week-cycle. Sintilimab (Innovent Biologics, Suzhou, China) was prescribed at a fixed dose of 200 mg every 3 weeks. 
Follow-up of the patients was conducted at a 3- to 6-week interval. Treatment was discontinued in cases of progressive disease confirmed by the follow-up CT or MR or in patients who had hepatic decompensation with ChildPugh C or clinical progression to ECOG performance status $>2$, or intolerable toxicity even after the dose adjustment of regorafenib (to $80 \mathrm{mg} / \mathrm{day}$ ) or treatment interruption. Patients who encountered intolerable toxicity in the regosintilimab group might discontinue one treatment of regorafenib or sintilimab and retained the other under the physician's suggestion. Patients with discontinuation of second-line treatment might receive post-line treatments, such as apatinib, and/or ICl immunotherapy other than sintilimab, and/or regional therapies, i.e. transarterial chemoembolization, or lodine125 seed implantation, for patients with Child-Pugh A/B and ECOG score 0-2, or best support treatment for patients with Child-Pugh C or ECOG score $>2$, according to the attending physicians' consensus.

\section{Outcomes}

Adverse events (AEs) related to treatment were recorded according to National Cancer Institute Common Terminology Criteria for Adverse Events, version 5.0 [18].

Treatment responses were assessed based on the dynamic CT or MR imaging, according to the Response Evaluation Criteria in Solid Tumors (RESICT) version 1.1 [19] and modified RESICT (mRESICT) [20]. ORR was defined as the incidence of complete response and partial response. Disease control rate (DCR) was defined as the incidence of complete response, partial response, and stable disease.

Progression-free survival (PFS) was defined as the time from the initiation of regorafenib until the date when tumor progression or death was confirmed or the last follow-up in censored data. OS was defined as the time from the initiation of regorafenib until death or the last follow-up in censored data.

\section{Statistical Analyses}

Categorical data was presented as number (percentage) and quantitative data as median value (Interquartile range [IQR]) unless otherwise indicated. Pearson $\chi 2$ test, correction of continuity, or Fisher's exact test were used to compare the categorical data of baseline patient characteristics, adverse events, and treatment responses between the two groups, as appropriate. Mann-Whitney $U$ test or Student's $t$ test were used to compare the quantitative data of baseline characteristics or mean dose of regorafenib between the two groups. Receiver operating characteristic (ROC) curve analyses were performed to determine the optimal cut-off values of lymphocyte, neutrophils, platelet, NLR, and PLR for predicting a 1-year survival. Curves of OS and PFS were determined by the Kaplan-Meier method and the log-rank test was used for comparisons. Univariate analyses and multivariate analyses of prognostic factors for OS and PFS were performed with Cox proportional hazard regression model. Variables with a $P$ value $<.10$ in the univariate analysis were included into the multivariate analysis. All tests were 2-sided and $P<.05$ was considered statistically significant. All statistical analyses were performed using SPSS Statistics, version 22.0 (SPSS, Chicago, II).

\section{Results}

\section{Study Population}

A total of 94 patients with advanced HCC who received rego-sintilimab or regorafenib as second-line treatment were assessed for eligibility during the study period. Eleven patients were excluded because they met the exclusion criteria 
(Fig. 1). Finally, 83 patients were included in this study (rego-sintilimab group, $n=48$; regorafenib group, $n=35$ ). The baseline characteristics between the two groups were not significantly different (Table 1).

In detail, $37.5 \%$ (18/48) of patients in rego-sintilimab group and 34.3\% (12/35) in regorafenib group had Child-Pugh B liver function $(P=.763) .41 .7 \%(20 / 48)$ of patients in rego-sintilimab group and $37.1 \%(13 / 35)$ in regorafenib group received lenvatinib in first-line treatment $(P=.678)$. Median time on first-line treatment was 8.0 months (IQR, 4.6-12.3) in rego-sintilimab group and 7.9 months (IQR, 5.2-13.7) in regorafenib group $(P=.941)$. Mean daily dose of regorafenib was $108.3 \mathrm{mg} \pm 23.3 \mathrm{in}$ rego-sintilimab group and $114.3 \mathrm{mg} \pm 24.0$ in regorafenib group $(P=.260)$. Median duration of second-line treatment with rego-sintilimab or regorafenib was 5.8 months (IQR, 3.5-9.4) in regosintilimab group and 3.9 months (IQR, 2.4-6.8) in regorafenib group.

\section{Safety}

Treatment-related AEs are shown in Table 2. No treatment-related mortality occurred. The overall incidence of AEs was similar between rego-sintilimab group and regorafenib group (any grade: $93.8 \%$ vs $89.6 \%, P=.661$; grade $3 / 4$ : $39.6 \%$ vs $31.4 \%, P=.445)$. There was a higher incidence of any grade rash in rego-sintilimab group than in regorafenib group $(22.9 \%$ vs $5.7 \%, P=.033)$. Incidences of other AEs were not significantly different between the two groups. AEs of any grade with an incidence over $20 \%$ in rego-sintilimab group included fatigue, hand-foot skin reaction, rash, hypertension, hypothyroidism, hyperbilirubinemia, diarrhea, and anorexia. Grade 3/4 AEs with an incidence over $5 \%$ in rego-sintilimab group included hand-foot skin reaction, hypertension, hyperbilirubinemia.

Treatment was discontinued because of $A E$ in $12.5 \%$ (6/48) of patients in rego-sintilimab group and in 8.6\% (3/35) in regorafenib group $(P=.833)$. Grade 4 skin toxicity was observed in 1 patient after 2 cycles of rego-sintilimab and grade 4 hyperbilirubinemia in 1 patient with immune-related hepatitis confirmed by liver biopsy after 6 cycles of regosintilimab, and both recovered from discontinuation of sintilimab and treatment with glucocorticoid. Other 4 cases of treatment discontinuation in rego-sintilimab group were caused by grade $3 \mathrm{AEs}$, i.e. rash in 1 patient, pneumonitis in 1 patient, and gastrointestinal hemorrhage in 2 patients.

\section{Treatment Response}

Tumor responses of the two groups were shown in Table 3. Two patients (5\%) in rego-sintilimab group versus no patients in regorafenib group had a complete response. The DCR for rego-sintilimab group was significantly higher than that observed in regorafenib group $(72.9 \%$ vs $48.6 \%$ by RECIST $1.1, P=.024 ; 72.9 \%$ vs $51.4 \%$ by mRECIST, $P=$ .044). The ORR for rego-sintilimab group was significantly higher than that observed in regorafenib group when assessed by mRECIST (33.3\% vs $14.3 \%, P=.049)$, but there was only a nonsignificant better ORR trend in regosintilimab group compared with regorafenib group when assessed by RECIST $1.1(22.9 \%$ vs $8.6 \%, P=.085)$.

\section{ROC curve analyses}

ROC curve analyses for predicting 1-year survival revealed that the optimal cut-off values for NLR, PLR, and neutrophils were 3.56 (area under the curve [AUC], .698; 95\% Cl, .579-.818; $P=.004$ ), 119 (AUC, .656; 95\% Cl, $.530-.782 ; P=.022$ ) and $4.53 * 10^{9} / \mathrm{L}$ (AUC .699; $95 \% \mathrm{Cl}, .575-.822 ; P=.003$ ), respectively. Thus, these variables were clarified into NLR $\leq 3.5 />3.5, \mathrm{PLR} \leq 120 />120$, and neutrophils $\leq 4.5^{\star} 10^{9} / \mathrm{L} />4.5^{\star} 10^{9} / \mathrm{L}$. The AUC for lymphocyte and platelet were not statistically significant $(P=0.121$ and 0.284 , respectively), and thus their cut-off values were not determined by the ROC curve.

\section{Progression-free survival}


The median follow-up duration was 12.8 months (IQR, 7.0-16.5) for the rego-sintilimab group and 8.7 months (IQR, 5.3-12.4) for the regorafenib group. During follow-up, 41 of 48 (85.4\%) patients in the rego-sintilimab group and 34 of $35(97.1 \%)$ patients in the regorafenib group experienced tumor progression. The median PFS in regosintilimab group vs in regorafenib group were 5.1 months $(95 \% \mathrm{Cl}, 3.4-6.8)$ vs 3.0 months $(95 \% \mathrm{Cl}, 2.3-3.7)$, respectively $(P=.001)$ (Fig. 2).

According to the uni- and multi-variate analyses, regorafenib alone (HR, 2.186; $95 \% \mathrm{Cl}, 1.353-3.532 ; P=.001)$, $\mathrm{a}$ fetoprotein (HR per $\left.10^{4} \mathrm{ng} / \mathrm{mL}, 1.044 ; 95 \% \mathrm{Cl}, 1.019-1.069 ; P=.001\right)$, and NLR > 3.5 (HR, 1.769; 95\% Cl, $1.093-$ 2.863; $P=.020$ ) were independent prognostic factors for PFS (Table 4).

\section{Overall survival}

During the follow-up period, 32 of 48 (66.7\%) patients in rego-sintilimab group and 27 of 35 (77.1\%) patients in regorafenib group died. The 6-, 12-, and 24-month OS rates were 83.3\%, 54.1\%, and 36.3\% (median OS, 13.3 months [95\% $\mathrm{Cl}, 9.5-17.1]$ ), respectively, in rego-sintilimab group and $76.2 \%, 31.4 \%$, and $0 \%$ (median 0 , 9.1 months [95\% $\mathrm{Cl}, 5.6-12.5])$, respectively, in regorafenib group $(P=.001)$ (Fig. 3). According to the uni- and multi-variate analyses, regorafenib alone (HR, 2.141; 95\% Cl, 1.178-3.890; $P=.012)$, Child-Pugh B liver function (HR, 2.225; $95 \%$ $\mathrm{Cl}, 1.301-3.804 ; P=.003)$, and NLR $>3.5(\mathrm{HR}, 1.897 ; 95 \% \mathrm{Cl}, 1.075-3.348 ; P=.027)$ were independent prognostic factors for OS (Table 5).

Subgroup analyses showed that, in patients with Child-Pugh A (16.4 vs 11.5 months; $P=.005)$ or B (8.8 vs 6.4 months; $P=.032$ ) liver function, the median OS was significantly improved in rego-sintilimab group compared with regorafenib group (Fig. $4 a$ and $4 b$ ). In patients with an NLR $\leq 3.5$, the median OS was significantly improved in rego-sintilimab group compared with regorafenib group (16.3 vs 11.5 months; $P=.012$ ), whereas it was not significantly different between the two groups in patients with an NLR > 3.5 (8.4 vs 7.0 months; $P=.288$ ) (Fig. 4c and 4d).

\section{Discussion}

Our study showed that rego-sintilimab conferred a significant survival benefit when compared with regorafenib in a second-line setting in advanced HCC patients who failed prior sorafenib or lenvatinib treatment. This finding was associated with an increase in median OS from 9.1 months to 13.3 months. In the multivariate analyses, combing sintilimab was an independent predictor for better OS and PFS. This survival benefit may be attributed to a higher ORR and DCR and a longer PFS in patients who received rego-sintilimab compared to those who received regorafenib alone. A recent phase $1 \mathrm{~b}$ trial investigated regorafenib combined with $\mathrm{ICI}$ pembrolizumab in first-line treatment of HCC and showed a promising ORR of 28\% by RECIST 1.1 [11], while the ORR by RECIST 1.1 in the regosintilimab group in our study was $22.9 \%$ in a second-line setting. These results indicated that rego-sintilimab had an advantage over regorafenib alone: regorafenib potently inhibited JAK1/2-STAT1 and MAPK signaling and subsequently attenuated the PD-L1 expression in the tumor [21], and increased intratumoral CD8 + T-cell infiltration through vasculature normalization by targeting VEGFR2/3 [12], which probably improve the efficacy of ICI sintilimab.

The median OS in the regorafenib group was 9.1 months, which seems to be poorer than the median OS of $10.3-$ 12.1 months for second-line regorafenib treatment in previous studies $[4,22,23]$. It was worth noting that these studies included only patients with Child-Pugh A liver function, while there were about 35\% of patients in our study had Child-Pugh B HCC. The OS of Child-Pugh B patients with regorafenib treatment was reported significantly poorer than the Child-Pugh A cohort in a multicentre study [24], which was consistent with the results in our study. 
Nonetheless, an effective treatment is still demanded for Child-Pugh B patients. Casadei's study showed that $35 \%$ of patients, who had Child-Pugh A HCC before sorafenib, might encountered a liver function deterioration to Child-Pugh $B$ when sorafenib ceased [25]. The latest phase I/II study by Kudo $\mathrm{M}$ et al. showed that ICl immunotherapy had favorable safety for patients with Child-Pugh B HCC, comparable to that seen in Child-Pugh A patients [26]. Similarly, our subgroup analyses indicated that rego-sintilimab improved patient survival for Child-Pugh B HCC was well as for Child-Pugh A HCC when compared to regorafenib alone.

An effective prognostic biomarker for immunotherapy in HCC patients is currently lacking and urgently needed [27]. NLR is an easily accessed and widely available blood-based clinical biomarker. A lower NLR may delineate a healthy host immune anti-tumor response [28], and was an independent predictor for PFS and OS in our study. Its clinical relevance is thought to be that an elevated peripheral neutrophil count is a marker of chronic inflammation which often leads to impaired immunity [29], whereas the peripheral lymphocyte count is a hallmark of a healthy cytotoxic T-cell response [30]. In our subgroup analyses, for HCC patients with an NLR > 3.5, rego-sintilimab might not confer a survival benefit compared to regorafenib alone. This result may help to select patients suitable for second-line treatment with rego-sintilimab.

The safety profile of rego-sintilimab was generally consistent with historical data on regorafenib [4] and sintilimab [13], with no new AEs reported. Combining sintilimab did not increase the overall incidence of any grade or grade 3/4 AEs on the basis of regorafenib treatment. Treatment in $12.5 \%$ of patients in rego-sintilimab group were discontinued because of AEs, which was similar to the incidence previously reported with sintilimab-bevacizumab biosimilar treatment (14\%) [13].

This study has several limitations. First, as a retrospective study, the comparison of rego-sintilimab and regorafenib may be subject to selection bias, and no matched pair analysis between the two groups was performed because of a small sample size. Instead, we conducted multivariate analyses and subgroup analyses to make a correction for confounding factors. Second, sample size in this study was relatively small. Results of the subgroup analyses should be cautiously interpreted and validation by further studies is needed. Third, the baseline NLR cut-off values are different (range 1.9-5.0) in each study [31]. This heterogeneity could hinder the application of NLR in the clinical setting. Thus, large cohort studies are required to establish the most appropriate NLR cut-off value in HCC patients with immunotherapy.

In conclusion, rego-sintilimab was tolerable and yielded promising outcomes in a second-line setting for patients with advanced HCC and Child-Pugh A or B liver function. These patients appeared to benefit from rego-sintilimab, and have better treatment responses, PFS, and OS, in comparison to regorafenib alone, especially in those who had an NLR $\leq 3.5$.

\section{Declarations}

Funding: This work was supported by the National Natural Science Foundation of China (Grant No. 82001930, 81873920), the Science and Technology Program of Guangzhou, China (Grant No. 202002030135), High-Level University Clinical Research Promotion Program of Guangzhou Medical University (No. B185004019), and the National Natural Science Foundation of China (Grant No. 81801809).

Conflict of interest: The authors declare that the research was conducted in the absence of any commercial or financial relationships that could be construed as a potential conflict of interest. 
Ethics approval: Appropriate Ethics Committee approval has been obtained for the research reported and the requirement to obtain informed consent for the study was waived.

Consent to participate: All patients were informed that regorafenib was standard second-line treatment and regorafenib combined with sintilimab was an alternative treatment. Moreover, all patients were informed of the economic cost and potential toxicity. Final treatment decisions were made by the patients.

Availability of data and material: All data generated or analyzed during this study are available by contacting the corresponding author.

\section{Authors' contributions:}

Jingjun Huang: conceptualization, methodology, validation, investigation, data curation, writing-original draft preparation, writing-review and editing, and funding acquisition. Yongjian Guo: conceptualization, methodology, validation, investigation, resources, data curation, writing-original draft preparation, writing-review and editing. Wensou Huang: conceptualization, methodology, validation, investigation, resources, data curation, writing-original draft preparation. Zining Xu: investigation, data curation, and writing-original draft preparation. Liteng Lin: investigation and writing-original draft preparation. Jingwen Zhou: investigation and writing-original draft preparation. Licong Liang: investigation and writing-review and editing. Yaqin Zhang: investigation and writingreview and editing. Juan Zhou: investigation and writing-review and editing. Mingyue Cai: methodology, formal analysis, investigation, writing-review and editing. Kangshun Zhu: conceptualization, writing-review and editing, supervision, project administration, and funding acquisition.

\section{References}

1. European Association for the Study of the Liver. Galle PR, Forner A, Llovet JM, Mazzaferro V, Piscaglia F, Raoul $\mathrm{JL}$, et al. EASL Clinical Practice Guidelines: Management of hepatocellular carcinoma. J Hepatol 2018; 69(1):182-236.

2. Llovet JM, Ricci S, Mazzaferro V, Hilgard P, Gane E, Blanc JF, et al. SHARP Investigators Study Group. Sorafenib in advanced hepatocellular carcinoma. N Engl J Med. 2008;359(4):378-90.

3. Kudo M, Finn RS, Qin S, Han KH, Ikeda K, Piscaglia F, et al. Lenvatinib versus sorafenib in first-line treatment of patients with unresectable hepatocellular carcinoma: a randomised Phase 3 non-inferiority trial. Lancet. 2018;391(10126):1163-73.

4. Bruix J, Qin S, Merle P, Granito A, Huang YH, Bodoky G, et al. RESORCE Investigators. Regorafenib for patients with hepatocellular carcinoma who progressed on sorafenib treatment (RESORCE): a randomised, double-blind, placebo-controlled, phase 3 trial. Lancet. 2017;389(10064):56-66.

5. Abou-Alfa GK, Meyer T, Cheng AL, El-Khoueiry AB, Rimassa L, Ryoo BY, et al. Cabozantinib in Patients with Advanced and Progressing Hepatocellular Carcinoma. N Engl J Med. 2018;379(1):54-63.

6. Zhu AX, Kang YK, Yen CJ, Finn RS, Galle PR, Llovet JM, et al. REACH-2 study investigators. Ramucirumab after sorafenib in patients with advanced hepatocellular carcinoma and increased a-fetoprotein concentrations (REACH-2): a randomised, double-blind, placebo-controlled, phase 3 trial. Lancet Oncol. 2019;20(2):282-96.

7. El-Khoueiry AB, Sangro B, Yau T, Crocenzi TS, Kudo M, Hsu C, et al. Nivolumab in patients with advanced hepatocellular carcinoma (CheckMate 040): an open-label, non-comparative, phase 1/2 dose escalation and expansion trial. Lancet. 2017;389(10088):2492-502. 
8. Zhu AX, Finn RS, Edeline J, Cattan S, Ogasawara S, Palmer D, et al. KEYNOTE-224 investigators. Pembrolizumab in patients with advanced hepatocellular carcinoma previously treated with sorafenib (KEYNOTE-224): a nonrandomised, open-label phase 2 trial. Lancet Oncol. 2018;19(7):940-52.

9. Finn RS, Ryoo BY, Merle P, Kudo M, Bouattour M, Lim HY, et al. KEYNOTE-240 investigators. Pembrolizumab As Second-Line Therapy in Patients With Advanced Hepatocellular Carcinoma in KEYNOTE-240: A Randomized, Double-Blind, Phase III Trial. J Clin Oncol. 2020;38(3):193-202.

10. Bangaru S, Marrero JA, Singal AG. Review article: new therapeutic interventions for advanced hepatocellular carcinoma. Aliment Pharmacol Ther. 2020;51(1):78-89.

11. El-Khoueiry AB, Kim RD, Harris WP, Sung MW, Waldschmidt D, Cabrera R, et al. Updated results of a phase 1b study of regorafenib (REG) $80 \mathrm{mg} /$ day or $120 \mathrm{mg}$ /day plus pembrolizumab (PEMBRO) for first-line treatment of advanced hepatocellular carcinoma (HCC). J Clin Oncol. 2021;39:15_suppl, 4078-8.

12. Shigeta K, Matsui A, Kikuchi H, Klein S, Mamessier E, Chen IX, et al. Regorafenib combined with PD1 blockade increases CD8 T-cell infiltration by inducing CXCL10 expression in hepatocellular carcinoma. J Immunother Cancer. 2020;8(2):e001435.

13. Ren Z, Xu J, Bai Y, Xu A, Cang S, Du C, et al. ORIENT-32 study group. Sintilimab plus a bevacizumab biosimilar (IBI305) versus sorafenib in unresectable hepatocellular carcinoma (ORIENT-32): a randomised, open-label, phase 2-3 study. Lancet Oncol. 2021;22(7):977-90.

14. Brahmer JR, Drake CG, Wollner I, Powderly JD, Picus J, Sharfman WH, et al. Phase I study of single-agent antiprogrammed death-1 (MDX-1106) in refractory solid tumors: safety, clinical activity, pharmacodynamics, and immunologic correlates. J Clin Oncol. 2010;28(19):3167-75.

15. Wang J, Fei K, Jing H, Wu Z, Wu W, Zhou S, et al. Durable blockade of PD-1 signaling links preclinical efficacy of sintilimab to its clinical benefit. MAbs. 2019;11(8):1443-51.

16. Marrero JA, Kulik LM, Sirlin CB, Zhu AX, Finn RS, Abecassis MM, et al. Diagnosis, Staging, and Management of Hepatocellular Carcinoma: 2018 Practice Guidance by the American Association for the Study of Liver Diseases. Hepatology. 2018;68(2):723-50.

17. Suner A, Carr BI. Platelet-to-lymphocyte and neutrophil-to-lymphocyte ratios predict tumor size and survival in HCC patients: Retrospective study. Ann Med Surg (Lond). 2020;58:167-71.

18. National Cancer Institute. Common terminology criteria for adverse events, version 5.0.

https://ctep.cancer.gov/protocolDevelopment/electronic_applications/ctc.htm\#ctc_50. Published November 27, 2017. Accessed December 16, 2020.

19. Eisenhauer EA, Therasse P, Bogaerts J, Schwartz LH, Sargent D, Ford R, et al. New response evaluation criteria in solid tumours: revised RECIST guideline (version 1.1). Eur J Cancer. 2009;45(2):228-47.

20. Lencioni R, Llovet JM. Modified RECIST (mRECIST) assessment for hepatocellular carcinoma. Semin Liver Dis. 2010;30(1):52-60.

21. Wu RY, Kong PF, Xia LP, Huang Y, Li ZL, Tang YY, et al. Regorafenib Promotes Antitumor Immunity via Inhibiting PD-L1 and ID01 Expression in Melanoma. Clin Cancer Res. 2019;25(14):4530-41.

22. Bekir Hacioglu M, Kostek O, Karabulut S, Tastekin D, Sezgin Goksu S, et al. Efficacy of regorafenib in the secondand third-line setting for patients with advanced hepatocellular carcinoma: A real life data of multicenter study from Turkey. J BUON. 2020;25(4):1897-903.

23. Yoo C, Byeon S, Bang Y, Cheon J, Kim JW, Kim JH, et al. Regorafenib in previously treated advanced hepatocellular carcinoma: Impact of prior immunotherapy and adverse events. Liver Int. 2020;40(9):2263-71. 
24. Kim HD, Bang Y, Lee MA, Kim JW, Kim JH, Chon HJ, et al. Regorafenib in patients with advanced Child-Pugh B hepatocellular carcinoma: A multicentre retrospective study. Liver Int. 2020;40(10):2544-52.

25. Casadei Gardini A, Frassineti GL, Foschi FG, Ercolani G, Ulivi P. Sorafenib and Regorafenib in HBV- or HCVpositive hepatocellular carcinoma patients: Analysis of RESORCE and SHARP trials. Dig Liver Dis. 2017;49(8):943-4.

26. Kudo M, Matilla A, Santoro A, Melero I, Gracián AC, Acosta-Rivera M, et al. CheckMate 040 cohort 5: A phase I/II study of nivolumab in patients with advanced hepatocellular carcinoma and Child-Pugh B cirrhosis. J Hepatol. 2021;75(3):600-9.

27. D'Alessio A, Cammarota A, Prete MG, Pressiani T, Rimassa L. The evolving treatment paradigm of advanced hepatocellular carcinoma: putting all the pieces back together. Curr Opin Oncol. 2021;33(4):386-94.

28. Ameratunga M, Chénard-Poirier M, Moreno Candilejo I, Pedregal M, Lui A, Dolling D, et al. Neutrophil-lymphocyte ratio kinetics in patients with advanced solid tumours on phase I trials of PD-1/PD-L1 inhibitors. Eur J Cancer. 2018;89:56-63.

29. Dumitru CA, Moses K, Trellakis S, Lang S, Brandau S. Neutrophils and granulocytic myeloid-derived suppressor cells: immunophenotyping, cell biology and clinical relevance in human oncology. Cancer Immunol Immunother. 2012;61(8):1155-67.

30. Nagai S, Abouljoud MS, Kazimi M, Brown KA, Moonka D, Yoshida A. Peritransplant lymphopenia is a novel prognostic factor in recurrence of hepatocellular carcinoma after liver transplantation. Transplantation. 2014;97(6):694-701.

31. Xiao WK, Chen D, Li SQ, Fu SJ, Peng BG, Liang LJ. Prognostic significance of neutrophil-lymphocyte ratio in hepatocellular carcinoma: a meta-analysis. BMC Cancer. 2014;14:117.

\section{Tables}

\section{Table 1 Baseline patient characteristics at initiation of second-line treatment}




\begin{tabular}{|c|c|c|c|}
\hline Characteristic & $\begin{array}{l}\text { Rego-sintilimab Group ( } \mathrm{N}= \\
\text { 48) }\end{array}$ & $\begin{array}{l}\text { Regorafenib Group ( }= \\
\text { 35) }\end{array}$ & $P$ value \\
\hline Male & $42(87.5)$ & $30(85.7)$ & $>.999$ \\
\hline Age (year) & $55(40-61)$ & $51(47-63)$ & .506 \\
\hline ECOG score 1 & $16(33.3)$ & $16(45.7)$ & .252 \\
\hline Positive for HBsAg & $40(83.3)$ & $32(91.4)$ & .455 \\
\hline Child-Pugh B & $18(37.5)$ & $12(34.3)$ & .763 \\
\hline Presence of ascites & $11(22.9)$ & $4(11.4)$ & 179 \\
\hline Tumor diameter (cm) & $5.9(4.2-8.8)$ & $5.5(4.3-7.0)$ & .901 \\
\hline$>3$ intrahepatic tumors & $33(68.8)$ & $25(71.4)$ & .793 \\
\hline Macrovascular invasion & $32(66.7)$ & $20(57.1)$ & .376 \\
\hline Extrahepatic metastasis & $30(62.5)$ & $21(60.0)$ & .817 \\
\hline Firs-line treatment with lenvatinib & $20(41.7)$ & $13(37.1)$ & .678 \\
\hline Time on first-line treatment (months) & $8.0(4.6-12.3)$ & $7.9(5.2-13.7)$ & .941 \\
\hline Previous treatment procedures & & & .877 \\
\hline Surgery or radical ablation & $11(22.9)$ & $9(25.7)$ & \\
\hline TACE or HAIC \& & $33(68.8)$ & $24(68.6)$ & \\
\hline Others ${ }^{\wedge}$ & $4(8.3)$ & $2(5.7)$ & \\
\hline \multicolumn{4}{|l|}{ Laboratory test } \\
\hline a-fetoprotein $(\mathrm{ng} / \mathrm{mL})$ & $924(22-15862)$ & $594(12-7630)$ & .625 \\
\hline PIVKA-X (ng/mL) & $1445(138-12512)$ & $1984(496-16625)$ & .150 \\
\hline Alanine aminotransferase (U/L) & $29(20-45)$ & $36(28-49)$ & .177 \\
\hline Aspartate aminotransferase (U/L) & $43(31-72)$ & $48(26-67)$ & .340 \\
\hline Total bilirubin (umol/L) & $12.7(8.7-17.7)$ & $15.5(9.7-23.7)$ & .156 \\
\hline Albumin (g/dL) & $35.4(31.3-39.4)$ & $35.9(32.5-40.1)$ & .678 \\
\hline Lymphocyte (109/L) & $1.02(.66-1.36)$ & $.80(.60-1.33)$ & .262 \\
\hline Neutrophils (109/L) & $2.63(2.13-3.62)$ & $2.95(2.41-3.84)$ & .612 \\
\hline Platelet $\left(10^{9} / \mathrm{L}\right)$ & $125(101-206)$ & $130(84-177)$ & .583 \\
\hline \multicolumn{4}{|l|}{$\begin{array}{l}\text { Inflammation-based prognostic } \\
\text { score }\end{array}$} \\
\hline NLR & $2.80(1.94-5.25)$ & $3.85(2.01-6.08)$ & . 184 \\
\hline PLR & $153(96-195)$ & $152(79-267)$ & .846 \\
\hline
\end{tabular}


Categorical data was presented as number (percentage) and quantitative data as median value (Interquartile range). rego-sintilimab = regorafenib combined with sintilimab, ECOG = Eastern Cooperative Oncology Group, HBsAg = hepatitis B surface antigen, TACE = transarterial chemoembolization, HAIC = hepatic arterial infusion chemotherapy, PIVKA-II = protein Induced by Vitamin K Absence or Antagonist-II, NLR = neutrophil-to-lymphocyte ratio, PLR = platelet-to-lymphocyte ratio.

\& TACE or HAIC without surgery or radical ablation

^ Including palliative ablation, radiotherapy, or lodine125 seed implantation, without surgery, radical ablation, TACE, or HAIC.

Table 2 Adverse events in the two groups 


\begin{tabular}{|c|c|c|c|c|c|c|}
\hline \multirow[t]{2}{*}{ Adverse events } & \multicolumn{3}{|l|}{ Any Grade } & \multicolumn{3}{|c|}{ Grade 3 or 4} \\
\hline & $\begin{array}{l}\text { Rego- } \\
\text { sintilimab } \\
\text { Group } \\
(\mathrm{N}=48)\end{array}$ & $\begin{array}{l}\text { Regorafenib } \\
\text { Group } \\
(\mathrm{N}=35)\end{array}$ & $\begin{array}{l}P \\
\text { value }\end{array}$ & $\begin{array}{l}\text { Rego- } \\
\text { sintilimab } \\
\text { Group } \\
(\mathrm{N}=48)\end{array}$ & $\begin{array}{l}\text { Regorafenib } \\
\text { Group } \\
(\mathrm{N}=35)\end{array}$ & $\begin{array}{l}P \\
\text { value }\end{array}$ \\
\hline Overall incidence & $45(93.8)$ & $31(88.6)$ & .661 & $19(39.6)$ & $11(31.4)$ & .445 \\
\hline Fatigue & $17(35.4)$ & $10(28.6)$ & .511 & 0 & $2(5.7)$ & .175 \\
\hline Hand-foot skin reaction & $14(29.2)$ & $12(34.3)$ & .620 & $3(6.3)$ & $5(14.3)$ & .396 \\
\hline Rash & $11(22.9)$ & $2(5.7)$ & .033 & $2(4.2)$ & 0 & .506 \\
\hline Pneumonitis & $6(12.5)$ & $1(2.9)$ & .230 & $2(4.2)$ & 0 & .506 \\
\hline Hypertension & $13(27.1)$ & $8(22.9)$ & .662 & $7(14.6)$ & $4(11.4)$ & .928 \\
\hline Hypothyroidism & $11(22.9)$ & $5(14.3)$ & .325 & 0 & 0 & - \\
\hline Hyperthyroidism & $4(8.3)$ & $1(2.9)$ & .570 & 0 & 0 & - \\
\hline Hyperbilirubinemia & $11(22.9)$ & $7(20.0)$ & .750 & $3(6.3)$ & $2(5.7)$ & .919 \\
\hline Increased GGT & $5(10.4)$ & $3(8.6)$ & $>.999$ & $1(2.1)$ & 0 & $>.999$ \\
\hline Increased AST & $9(18.8)$ & $5(14.3)$ & .650 & $2(4.2)$ & $2(5.7)$ & $>.999$ \\
\hline Increased ALT & $6(12.5)$ & $3(8.6)$ & .833 & $1(2.1)$ & $1(2.9)$ & $>.999$ \\
\hline Leukopenia & $6(12.5)$ & $2(5.7)$ & .511 & $2(4.2)$ & 0 & .506 \\
\hline Lymphopenia & $5(10.4)$ & $2(5.7)$ & .718 & 0 & 0 & - \\
\hline Neutropenia & $4(8.3)$ & $4(11.4)$ & .924 & $1(2.1)$ & 0 & $>.999$ \\
\hline Thrombocytopenia & $5(10.4)$ & $4(11.4)$ & $>.999$ & $1(2.1)$ & $1(2.9)$ & $>.999$ \\
\hline Anemia & $6(12.5)$ & $2(5.7)$ & .511 & $1(2.1)$ & 0 & $>.999$ \\
\hline Proteinuria & $3(6.3)$ & $5(14.3)$ & .396 & 0 & 0 & - \\
\hline Increased creatinine & $1(2.1)$ & $1(2.9)$ & $>.999$ & 0 & 0 & - \\
\hline Diarrhea & $13(27.1)$ & $7(20.0)$ & .456 & $2(4.2)$ & $1(2.9)$ & $>.999$ \\
\hline Nausea & $5(10.4)$ & $4(11.4)$ & $>.999$ & 0 & 0 & - \\
\hline Anorexia & $12(25.0)$ & $8(22.9)$ & .822 & 0 & $1(2.9)$ & .429 \\
\hline Weight loss & $2(4.2)$ & $3(8.6)$ & .715 & 0 & 0 & - \\
\hline Constipation & $3(6.3)$ & $2(5.7)$ & .919 & 0 & 0 & - \\
\hline Presence of ascites & $1(2.1)$ & $1(2.9)$ & $>.999$ & 0 & 0 & - \\
\hline Gastrointestinal hemorrhage & $2(4.2)$ & $2(5.7)$ & $>.999$ & $2(4.2)$ & $2(5.7)$ & $>.999$ \\
\hline Gingival bleeding & $3(6.3)$ & $4(11.4)$ & .661 & 0 & 0 & - \\
\hline Epistaxis & 0 & $1(2.9)$ & .437 & 0 & 0 & - \\
\hline
\end{tabular}




\begin{tabular}{|lllllll|} 
Hoarseness & $5(10.4)$ & $3(8.6)$ & $>.999$ & 0 & 0 & - \\
\hline Infusion reaction & $4(8.3)$ & - & - & 0 & - & - \\
\hline
\end{tabular}

Data are numbers of patients and data in parentheses are percentages. rego-sintilimab = regorafenib combined with sintilimab, GGT = $y$ glutamyltransferase, AST = aspartate aminotransferase, ALT = alanine aminotransferase.

\section{Table 3 Treatment responses}

\begin{tabular}{|lllllll|}
\hline & mRECIST & & & RECIST 1.1 & \\
\hline Treatment response & $\begin{array}{l}\text { Rego- } \\
\text { sintilimab } \\
\text { Group } \\
(\mathbf{N}=48)\end{array}$ & $\begin{array}{l}\text { Regorafenib } \\
\text { Group } \\
(\mathrm{N}=35)\end{array}$ & $P$ value & $\begin{array}{l}\text { Rego- } \\
\text { sintilimab } \\
\text { Group } \\
(\mathbf{N}=48)\end{array}$ & $\begin{array}{l}\text { Regorafenib } \\
\text { Group } \\
(\mathrm{N}=35)\end{array}$ & $P$ value \\
\hline CR & 2 & 0 & $\ldots$ & 0 & 0 & $\ldots$ \\
\hline PR & 14 & 5 & $\ldots$ & 11 & 3 & $\ldots$ \\
\hline SD & 19 & 13 & $\ldots$ & 24 & 14 & $\ldots$ \\
\hline PD & 13 & 17 & $\ldots$ & 13 & 18 & $\ldots$ \\
\hline ORR, \% & 33.3 & 14.3 & .049 & 22.9 & 8.6 & .085 \\
\hline DCR, \% & 72.9 & 51.4 & .044 & 72.9 & 48.6 & .024 \\
\hline
\end{tabular}

Objective response rate $(\mathrm{ORR})=(\mathrm{CR}+\mathrm{PR}) / N$, and Disease control rate $(\mathrm{DCR})=(\mathrm{CR}+\mathrm{PR}+\mathrm{SD}) / N$, where $\mathrm{CR}$ is number of patients with complete response, $\mathrm{PR}$ is number of patients with partial response, $\mathrm{SD}$ is number of patients with stable disease, and $N$ is total number of patients. $\mathrm{PD}=$ progressive disease, rego-sintilimab $=$ regorafenib combined with sintilimab, RESICT = response evaluation criteria in solid tumors, $\mathrm{mRESICT}=$ modified RESICT .

Table 4 Analyses of prognostic factors for progression-free survival 


\begin{tabular}{|c|c|c|c|c|}
\hline \multirow[t]{2}{*}{ Factor } & \multicolumn{2}{|l|}{ Univariable analysis } & \multicolumn{2}{|l|}{ Multivariate analysis } \\
\hline & $\mathrm{HR}(95 \% \mathrm{Cl})$ & $P$ Value & $\mathrm{HR}(95 \% \mathrm{Cl})$ & $P$ Value \\
\hline Regorafenib alone & $2.188(1.361-3.518)$ & .001 & $2.186(1.353-3.532)$ & .001 \\
\hline Male & $1.296(.661-2.539)$ & .450 & & \\
\hline$\geq 65$ years & $.701(.346-1.419)$ & .324 & & \\
\hline ECOG score $=1$ & $1.142(.704-1.854)$ & .591 & & \\
\hline Positive for $\mathrm{HbsAg}$ & $.782(.374-1.636)$ & .513 & & \\
\hline Child-Pugh B & $.930(.567-1.525)$ & .774 & & \\
\hline Presence of ascites & $.903(.494-1.649)$ & .740 & & \\
\hline Tumor diameter (cm) & $1.168(.721-1.891)$ & .528 & & \\
\hline$>3$ intrahepatic tumors & $1.606(.963-2.678)$ & .069 & $\ldots$ & 192 \\
\hline Macrovascular invasion & $.854(.530-1.376)$ & .516 & & \\
\hline Extrahepatic metastasis & $1.558(.965-2.513)$ & .070 & $\ldots$ & .153 \\
\hline Firs-line treatment with lenvatinib & $.905(.526-1.154)$ & .174 & & \\
\hline Time on first-line treatment (month) & $1.008(.995-1.021)$ & .215 & & \\
\hline Previous surgery or ablation & $.576(.291-1.141)$ & .114 & & \\
\hline a-fetoprotein $\left(10^{4} \mathrm{ng} / \mathrm{mL}\right)$ & $1.047(1.023-1.072)$ & $<.001$ & $1.044(1.019-1.069)$ & .001 \\
\hline PIVKA-X $\left(10^{4} \mathrm{mAU} / \mathrm{mL}\right)$ & $1.077(1.017-1.141)$ & .011 & $\ldots$ & .272 \\
\hline Alanine aminotransferase $>50 \mathrm{U} / \mathrm{L}$ & $1.260(.730-2.176)$ & .407 & & \\
\hline Aspartate aminotransferase $>40 \mathrm{U} / \mathrm{L}$ & $1.036(.654-1.641)$ & .881 & & \\
\hline Total bilirubin $>22 \mathrm{umol} / \mathrm{L}$ & $1.231(.691-2.195)$ & .480 & & \\
\hline Albumin $<35 \mathrm{~g} / \mathrm{dL}$ & $.998(.626-1.590)$ & .993 & & \\
\hline Lymphocyte $<1.0 * 10^{9} / \mathrm{L}$ & $1.588(.996-2.530)$ & .052 & & .810 \\
\hline Neutrophils $>4.5^{\star} 10^{9} / \mathrm{L}$ & $1.908(1.001-3.637)$ & .050 & & .243 \\
\hline Platelet of $100-200^{*} 10^{9} / \mathrm{L}$ & Ref & & & \\
\hline Platelet $<100 * 10^{9} / \mathrm{L}$ & $1.899(.923-3.905)$ & .081 & & .591 \\
\hline Platelet $>200 * 10^{9} / \mathrm{L}$ & $1.877(.945-3.725)$ & .072 & $\ldots$ & .144 \\
\hline NLR $>3.5$ & $1.979(1.241-3.154)$ & .004 & $1.769(1.093-2.863)$ & .020 \\
\hline PLR $>120$ & $1.528(.939-2.489)$ & .088 & & .326 \\
\hline
\end{tabular}

The uni- and multi-variate analyses were performed using Cox proportional hazard regression model. $\mathrm{HR}=$ Hazard Ratio, $\mathrm{Cl}=$ confidence interval, ECOG = Eastern Cooperative Oncology Group, HBsAg = hepatitis B surface antigen, 
PIVKA-II = protein Induced by Vitamin K Absence or Antagonist-II, NLR = neutrophil-to-lymphocyte ratio, PLR = platelet-to-lymphocyte ratio.

\section{Table 5 Analyses of prognostic factors for overall survival}




\begin{tabular}{|c|c|c|c|c|}
\hline \multirow[t]{2}{*}{ Factor } & \multicolumn{2}{|l|}{ Univariable analysis } & \multicolumn{2}{|c|}{ Multivariate analysis } \\
\hline & $\mathrm{HR}(95 \% \mathrm{Cl})$ & $P$ Value & $\mathrm{HR}(95 \% \mathrm{Cl})$ & $P$ Value \\
\hline Regorafenib alone & $\begin{array}{l}2.481(1.434- \\
4.293)\end{array}$ & .001 & $\begin{array}{l}2.141(1.178- \\
3.890)\end{array}$ & .012 \\
\hline Male & $1.073(.525-2.193)$ & .846 & & \\
\hline$\geq 65$ years & $1.245(.561-2.765)$ & .590 & & \\
\hline ECOG score $=1$ & $\begin{array}{l}2.036(1.218- \\
3.403)\end{array}$ & .007 & $\ldots$ & .309 \\
\hline Positive for HbsAg & $.734(.314-1.713)$ & .474 & & \\
\hline Child-Pugh B & $\begin{array}{l}1.977(1.169- \\
3.346)\end{array}$ & .011 & $\begin{array}{l}2.225(1.301- \\
3.804)\end{array}$ & .003 \\
\hline Presence of ascites & $1.155(.609-2.188)$ & .659 & & \\
\hline Tumor diameter (per cm) & $1.037(.956-1.124)$ & .384 & & \\
\hline$>3$ intrahepatic tumors & $1.735(.960-3.136)$ & .068 & $\ldots$ & .116 \\
\hline Macrovascular invasion & $1.091(.639-1.863)$ & .750 & & \\
\hline Extrahepatic metastasis & $1.490(.847-2.620)$ & .167 & & \\
\hline Firs-line treatment with lenvatinib & $.756(.445-1.283)$ & .300 & & \\
\hline $\begin{array}{l}\text { Time on first-line treatment (per } \\
\text { month) }\end{array}$ & $.991(.970-1.012)$ & .385 & & \\
\hline Previous surgery or ablation & $.577(.291-1.142)$ & .114 & & \\
\hline a-fetoprotein (per $10^{4} \mathrm{ng} / \mathrm{mL}$ ) & $1.017(.997-1.038)$ & .095 & $\ldots$ & .811 \\
\hline PIVKA-X (per $\left.10^{4} \mathrm{mAU} / \mathrm{mL}\right)$ & $\begin{array}{l}1.059(1.005- \\
1.115)\end{array}$ & .031 & $\ldots$ & .983 \\
\hline Alanine aminotransferase $>50 \mathrm{U} / \mathrm{L}$ & $1.475(.827-2.630)$ & .188 & & \\
\hline Aspartate aminotransferase $>40 \mathrm{U} / \mathrm{L}$ & $\begin{array}{l}2.225(1.297- \\
3.816)\end{array}$ & .004 & $\ldots$ & .116 \\
\hline Total bilirubin $>22$ umol/L & $\begin{array}{l}1.898(1.044- \\
3.451)\end{array}$ & .036 & $\ldots$ & .471 \\
\hline Albumin $<35 \mathrm{~g} / \mathrm{dL}$ & $1.538(.920-2.570)$ & .100 & & \\
\hline Lymphocyte $<1.0^{*} 10^{9} / \mathrm{L}$ & $\begin{array}{l}1.920(1.113- \\
3.312)\end{array}$ & .019 & $\ldots$ & .672 \\
\hline Neutrophils $>4.5^{*} 10^{9} / \mathrm{L}$ & $\begin{array}{l}2.620(1.318- \\
5.209)^{-}\end{array}$ & .006 & & .176 \\
\hline Platelet of $100-200 * 10^{9} / L$ & Ref & & & \\
\hline Platelet $<100 * 10^{9} / \mathrm{L}$ & $\begin{array}{l}2.135(1.054- \\
4.327)\end{array}$ & .035 & & .682 \\
\hline Platelet $>200 * 10^{9} / \mathrm{L}$ & $\begin{array}{l}2.136(1.051- \\
4.343)\end{array}$ & .036 & & .624 \\
\hline
\end{tabular}


$\mathrm{NLR}>3.5$

PLR $>120$
2.406 (1.420-

$$
\text { 4.078) }
$$

1.910 (1.061-

3.440 )
.001

The uni- and multi-variate analyses were performed using Cox proportional hazard regression model. HR $=\mathrm{Hazard}$ Ratio, $\mathrm{Cl}$ = confidence interval, $\mathrm{ECOG}=$ Eastern Cooperative Oncology Group, $\mathrm{HBsAg}=$ hepatitis $\mathrm{B}$ surface antigen, PIVKA-II = protein Induced by Vitamin K Absence or Antagonist-II, NLR = neutrophil-to-lymphocyte ratio, PLR = platelet-to-lymphocyte ratio.

\section{Figures}

Advanced HCC treated with rego-sintilimab or regorafenib in a second-line setting (01/2019-12/2020)

Assessed for eligibility $(\mathrm{n}=94)$

HCC treated with rego-sintilimab

$$
(\mathrm{n}=54)
$$

\section{Excluded $(n=6)$}

- Previous apatinib treatment $(\mathrm{n}=2)$

- Previous immunotherapy $(n=2)$

- International normalized ratio $\geq 1.5(\mathrm{n}=1)$

- Follow up less than three months $(n=1)$

Advanced HCC treated with rego-sintilimab in a second-line setting $(n=48)$
HCC treated with regorafenib

$$
(n=40)
$$

Excluded $(\mathrm{n}=5)$

- Previous immunotherapy $(\mathrm{n}=2)$

- Other malignant tumor $(\mathrm{n}=1)$

- Medical comorbidities $(n=1)$

- Follow up less than three months $(n=1)$

Advanced HCC treated with regorafenib in a second-line setting $(n=35)$

Figure 1

Flow diagram shows exclusion in patients with advanced hepatocellular carcinoma (HCC) who receive regorafenib combined with sintilimab (rego-sintilimab) or regorafenib in a second-line setting. 


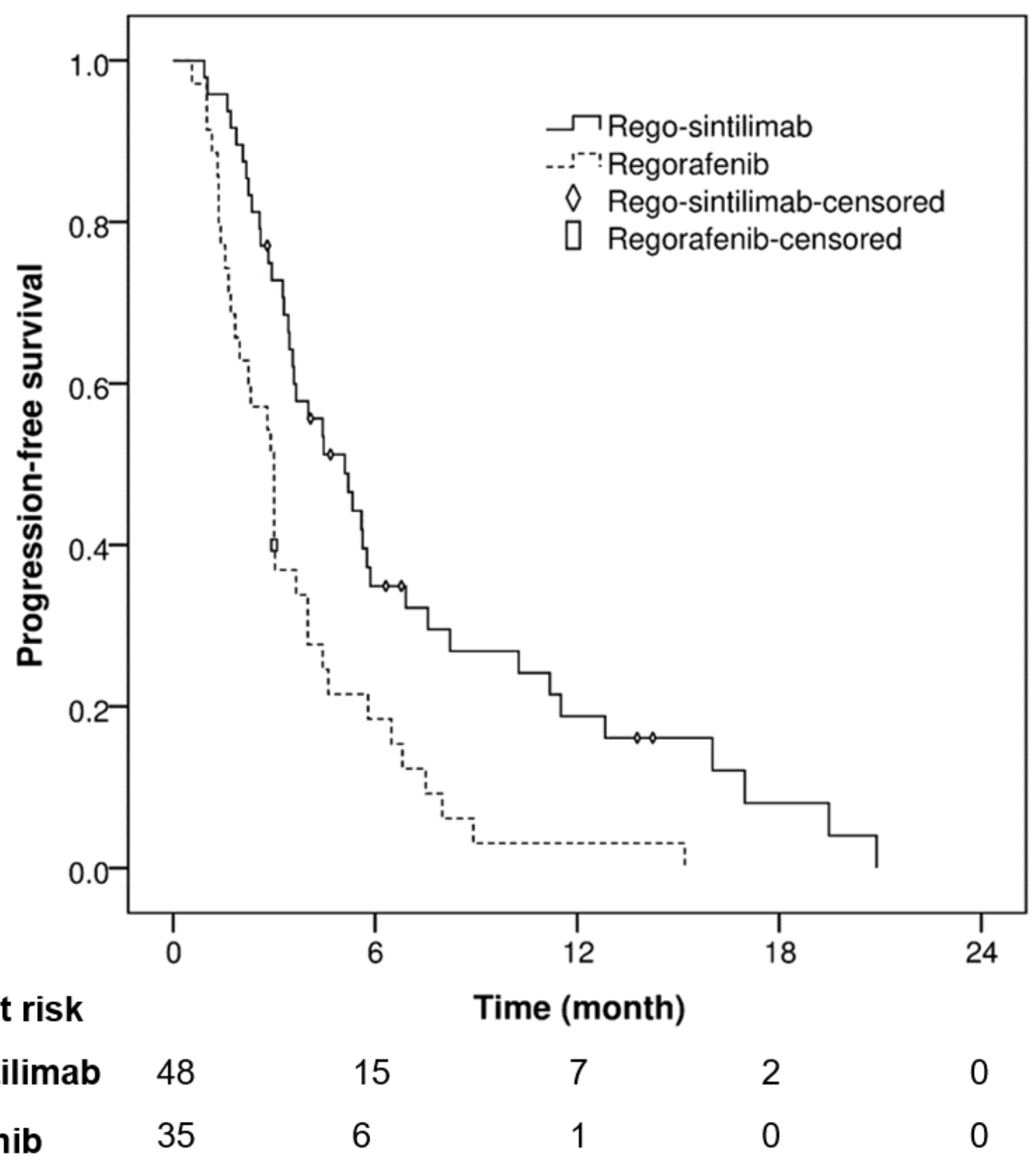

Figure 2

Kaplan-Meier curves of progression-free survival (PFS) for patients with advanced hepatocellular carcinoma who receive regorafenib combined with sintilimab (rego-sintilimab) (median PFS, 5.1 months; $95 \% \mathrm{Cl}, 3.4-6.8$ ) or regorafenib (median PFS, 3.0 months; $95 \% \mathrm{Cl}, 2.3-3.7 ; \mathrm{P}=.001$ ) in a second-line setting. $\mathrm{Cl}=$ confidence interval . 


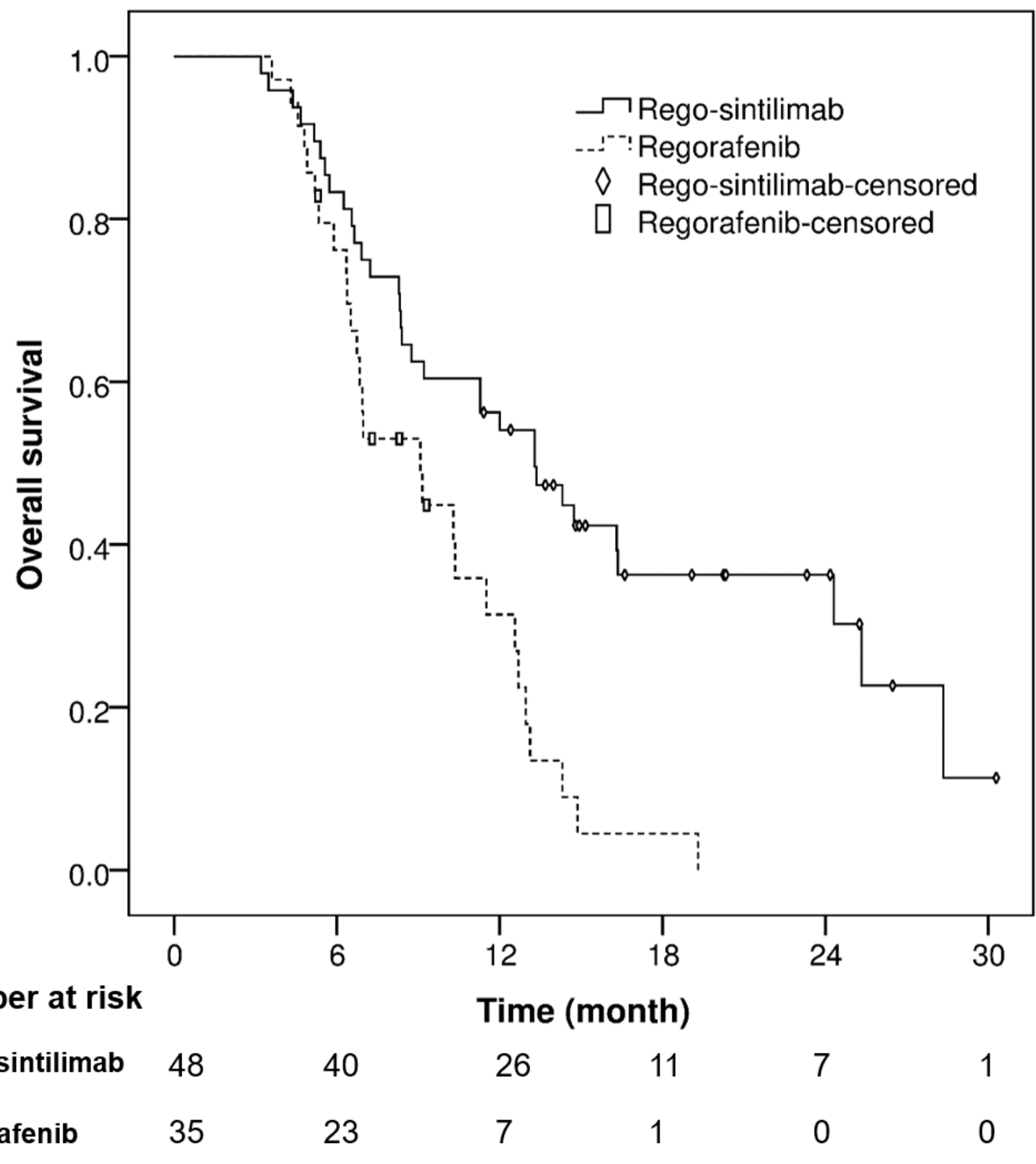

Figure 3

Kaplan-Meier curves of overall survival (OS) for patients with advanced hepatocellular carcinoma who receive regorafenib combined with sintilimab (rego-sintilimab) (median OS, 13.3 months; $95 \% \mathrm{Cl}$, 9.5-17.1) or regorafenib (median OS, 9.1 months; $95 \% \mathrm{Cl}, 5.6-12.5 ; \mathrm{P}=.001$ ) in a second-line setting. $\mathrm{Cl}=$ confidence interval. 

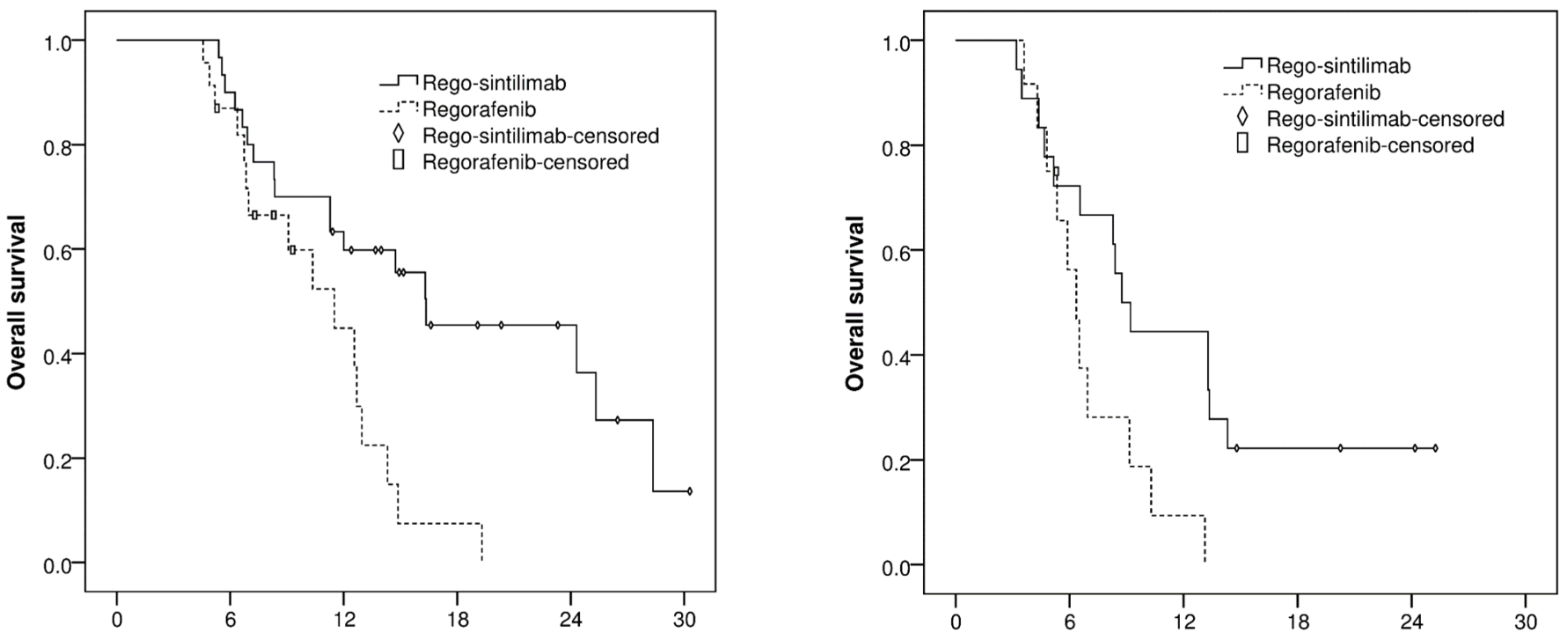

Number at risk

$\begin{array}{lllllll}\text { Rego-sintilimab } & 30 & 27 & 18 & 8 & 5 & 1 \\ \text { Regorafenib } & 23 & 17 & 6 & 1 & 0 & 0\end{array}$

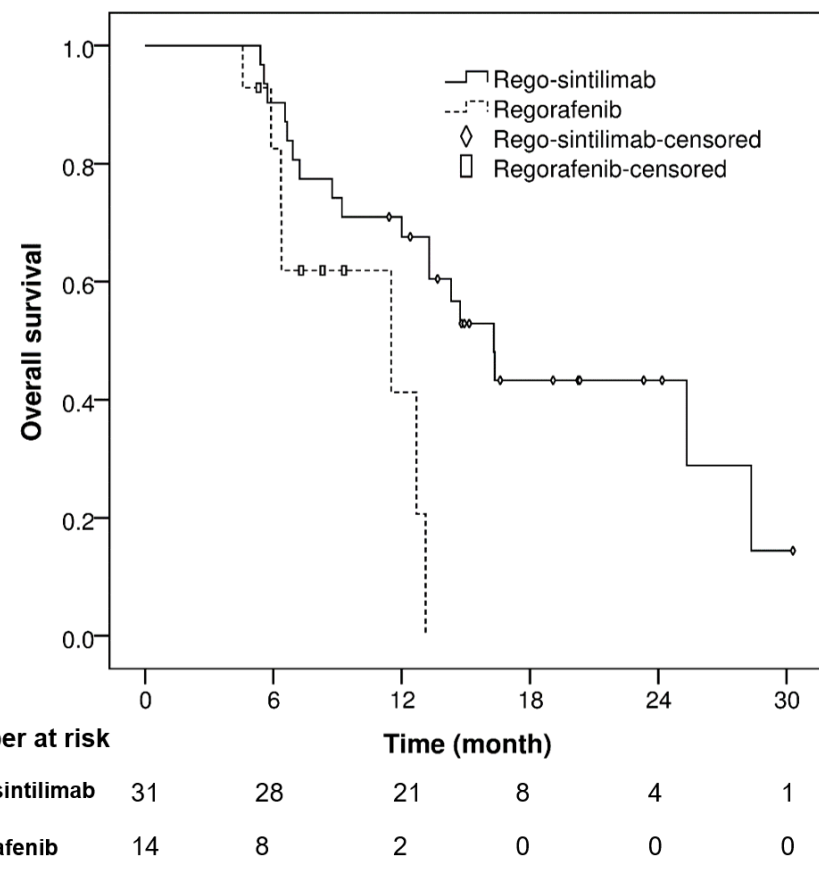

Number at risk

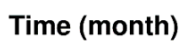

$\begin{array}{lllllll}\text { Rego-sintilimab } & 18 & 13 & 8 & 3 & 2 & 0 \\ \text { Regorafenib } & 12 & 6 & 1 & 0 & 0 & 0\end{array}$

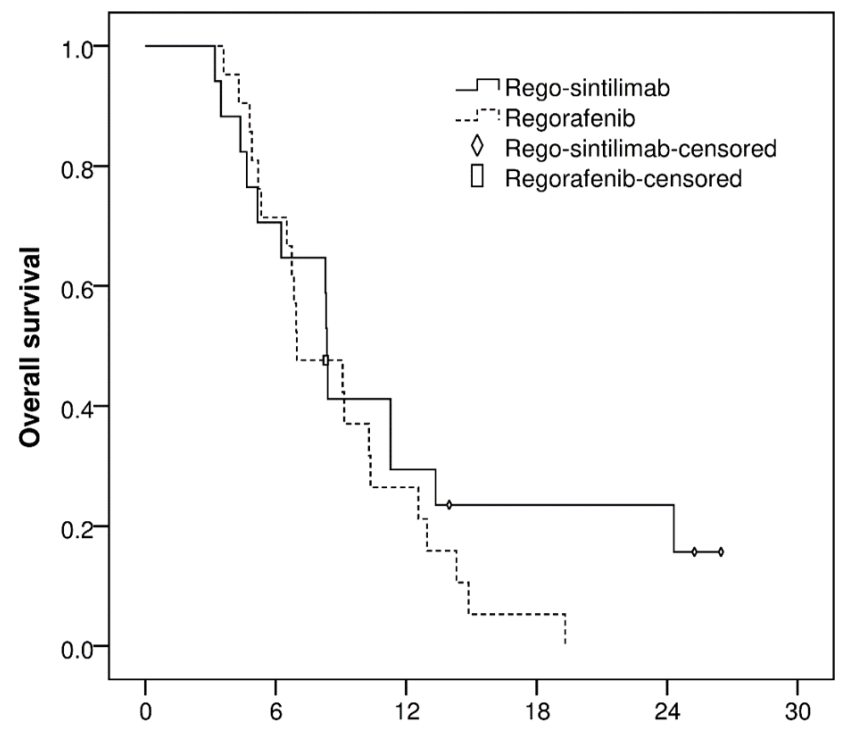

Number at risk

Time (month)

$\begin{array}{lllllll}\text { Rego-sintilimab } & 17 & 12 & 5 & 3 & 3 & 0 \\ \text { Regorafenib } & 21 & 15 & 5 & 1 & 0 & 0\end{array}$

\section{Figure 4}

Kaplan-Meier curves of overall survival (OS) for (a) Child-Pugh A patients (rego-sintilimab group: median OS, 16.4 months [95\% Cl, 6.6-26.1]; regorafenib group: median OS, 11.5 months [95\% Cl, 7.5-15.5]; $\mathrm{P}=.005$ ), (b) Child-Pugh B patients (rego-sintilimab group: median OS, 8.8 months [95\% $\mathrm{Cl}, 7.0-10.5]$; regorafenib group: median OS, 6.4 months [95\% Cl, 5.4-7.3]; $\mathrm{P}=.027$ ), (c) patients with NLR $\leq 3.5$ (rego-sintilimab group: median OS, 16.3 months [95\% Cl, 13.4-19.2]; regorafenib group: median OS, 11.5 months [95\% Cl, 1.9-21.1]; $\mathrm{P}=.012$ ), and (d) patients with NLR > 3.5 (rego-sintilimab group: median OS, 8.4 months [95\% Cl, 8.2-8.5]; regorafenib group: median OS, 7.0 months [95\% Cl, 3.7-10.2]; $\mathrm{P}=.274) . \mathrm{Cl}=$ confidence interval, NLR = neutrophil-to-lymphocyte ratio. 